\title{
Sandwich-Type Theorems for a Class of Multiplier Transformations Associated with the Noor Integral Operators
}

\author{
Nak Eun Cho' ${ }^{1}$ and Khalida Inayat Noor ${ }^{2}$ \\ ${ }^{1}$ Department of Applied Mathematics, Pukyong National University, Busan 608-737, Republic of korea \\ ${ }^{2}$ Department of Mathematics, COMSATS Institute of Information Technology, Islamabad 44000, Pakistan \\ Correspondence should be addressed to Nak Eun Cho, necho@pknu.ac.kr
}

Received 23 September 2011; Accepted 4 November 2011

Academic Editor: Muhammad Aslam Noor

Copyright (c) 2012 N. E. Cho and K. I. Noor. This is an open access article distributed under the Creative Commons Attribution License, which permits unrestricted use, distribution, and reproduction in any medium, provided the original work is properly cited.

We obtain some subordination- and superordination-preserving properties for a class of multiplier transformations associated with Noor integral operators defined on the space of normalized analytic functions in the open unit disk. The sandwich-type theorems for these transformations are also considered.

\section{Introduction}

Let $\mathscr{H}=\mathscr{H}(\mathbb{U})$ denote the class of analytic functions in the open unit disk $\mathbb{U}=\{z \in \mathbb{C}:|z|<1\}$. For $a \in \mathbb{C}$ and nonnegative integer $n$, let

$$
\mathscr{H}[a, n]=\left\{f \in \mathscr{H}: f(z)=a+a_{n} z^{n}+a_{n+1} z^{n+1}+\cdots\right\} .
$$

We also denote $\mathscr{A}$ by the subclass of $\mathscr{L}[a, 1]$ with the usual normalization $f(0)=f^{\prime}(0)-1=0$.

Let $f$ and $F$ be members of $\mathcal{H}$. The function $f$ is said to be subordinate to $F$, or $F$ is said to be superordinate to $f$, if there exists a function $w$ analytic in $\mathbb{U}$, with $w(0)=0$ and $|w(z)|<1$, and such that $f(z)=F(w(z))$. In such a case, we write $f<F$ or $f(z)<F(z)$. If the function $F$ is univalent in $\mathbb{U}$, then we have $f \prec F$ if and only if $f(0)=F(0)$ and $f(\mathbb{U}) \subset F(\mathbb{U})$ (cf. [1]).

Definition 1.1 (see [1]). Let $\phi: \mathbb{C}^{2} \rightarrow \mathbb{C}$ and let $h$ be univalent in $\mathbb{U}$. If $p$ is analytic in $\mathbb{U}$ and satisfies the differential subordination:

$$
\phi\left(p(z), z p^{\prime}(z)\right) \prec h(z) \quad(z \in \mathbb{U})
$$


then $p$ is called a solution of the differential subordination. The univalent function $q$ is called a dominant of the solutions of the differential subordination, or more simply a dominant if $p \prec q$ for all $p$ satisfying (1.2). A dominant $\tilde{q}$ that satisfies $\tilde{q} \prec q$ for all dominants $q$ of (1.2) is said to be the best dominant.

Definition 1.2 (see [2]). Let $\varphi: \mathbb{C}^{2} \rightarrow \mathbb{C}$ and let $h$ be analytic in $\mathbb{U}$. If $p$ and $\varphi\left(p(z), z p^{\prime}(z)\right)$ are univalent in $\mathbb{U}$ and satisfy the differential superordination:

$$
h(z) \prec \varphi\left(p(z), z p^{\prime}(z)\right) \quad(z \in \mathbb{U}),
$$

then $p$ is called a solution of the differential superordination. An analytic function $q$ is called a subordinant of the solutions of the differential superordination, or more simply a subordinant if $q \prec p$ for all $p$ satisfying (1.3). A univalent subordinant $\tilde{q}$ that satisfies $q \prec \tilde{q}$ for all subordinants $q$ of (1.3) is said to be the best subordinant.

Definition 1.3 (see [2]). We denote by $\mathcal{Q}$ the class of functions $f$ that are analytic and injective on $\overline{\mathbb{U}} \backslash E(f)$, where

$$
E(f)=\left\{\zeta \in \partial \mathbb{U}: \lim _{z \rightarrow \zeta}=\infty\right\}
$$

and are such that $f^{\prime}(\zeta) \neq 0$ for $\zeta \in \partial \mathbb{U} \backslash E(f)$.

Following Komatu [3], we introduce the integral operator $\mathcal{L}^{\lambda}(c): \mathcal{A} \rightarrow \mathcal{A}$ defined by

$$
\mathcal{L}^{\lambda}(c) f(z):=\frac{c^{\lambda}}{\Gamma(\lambda)} \int_{0}^{1} t^{c-2}\left(\log \frac{1}{t}\right)^{\lambda-1} f(t z) d t \quad(\operatorname{Re}\{c\}>0 ; \lambda \geq 0 ; f \in \mathcal{A}),
$$

where the symbol $\Gamma$ stands the Gamma function. We also note that the operator $\mathcal{L}^{\lambda}(c) f(z)$ defined by (1.5) can be expressed by the series expansion as follows:

$$
\mathcal{L}^{\lambda}(c) f(z)=z+\sum_{k=2}^{\infty}\left(\frac{c}{c+k-1}\right)^{\lambda} a_{k} z^{k}
$$

Obviously, we have, for $\lambda, v \geq 0$,

$$
\mathcal{L}^{\lambda}(c)\left(\mathcal{L}^{v}(c) f(z)\right)=\mathcal{L}^{\lambda+v}(c) f(z) .
$$

In particular, the operator $\mathcal{L}^{\lambda}(2)$ is closely related to the multiplier transformation studied earlier by Flett [4]. Various interesting properties of the operator $\mathcal{L}^{\lambda}(2)$ have been studied by Jung et al. [5] and Liu [6]. We also note from (1.6) that we can define the operator $\mathcal{L}^{\lambda}(c)$ for any real number $\lambda$.

Let

$$
f_{c}^{\curlywedge}(z)=z+\sum_{k=2}^{\infty}\left(\frac{c}{c+k-1}\right)^{\curlywedge} z^{k} \quad(\operatorname{Re}\{c\}>0 ; \lambda \geq 0)
$$


and let $f_{c}^{\lambda, \mu}$ be defined such that

$$
f_{c}^{\lambda}(z) * f_{c}^{\lambda, \mu}(z)=\frac{z}{(1-z)^{\mu}} \quad(\mu>0 ; z \in \mathbb{U})
$$

where the symbol $*$ stands for the Hadamard product (or convolution). Then, motivated essentially by the Noor integral operator [7] (see also [8-11]), we now introduce the operator $I_{\lambda, \mu}^{\kappa}: \mathcal{A} \rightarrow \mathcal{A}$, which are defined here by

$$
I_{c}^{\lambda, \mu} f(z)=\left(f_{c}^{\lambda, \mu} * f\right)(z) \quad(f \in \mathcal{A} ; \operatorname{Re}\{c\}>0 ; \lambda \geq 0 ; \mu>0)
$$

In view of (1.9) and (1.10), we obtain the following relations:

$$
\begin{aligned}
& z\left(I_{c}^{\lambda, \mu} f(z)\right)^{\prime}=c I_{c}^{\lambda+1, \mu} f(z)-(c-1) I_{c}^{\lambda, \mu} f(z), \\
& z\left(I_{c}^{\lambda, \mu} f(z)\right)^{\prime}=\mu I_{c}^{\lambda, \mu+1} f(z)-(\mu-1) I_{c}^{\lambda, \mu} f(z) .
\end{aligned}
$$

Making use of the principle of subordination between analytic functions, Miller et al. [12] investigated some subordination theorems involving certain integral operators for analytic functions in $\mathbb{U}$ (see, also [13]). Moreover, Miller and Mocanu [2] considered differential superordinations, as the dual concept of differential subordinations (see also [14]). In the present paper, we obtain the subordination- and superordination-preserving properties of the multiplier transformations $I_{c}^{\lambda, \mu}$ defined by (1.10) with the sandwich-type theorems.

The following lemmas will be required in our present investigation.

Lemma 1.4 (see [15]). Suppose that the function $H: \mathbb{C}^{2} \rightarrow \mathbb{C}$ satisfies the condition:

$$
\operatorname{Re}\{H(\text { is }, t)\} \leq 0,
$$

for all real $s$ and $t \leq-n\left(1+s^{2}\right) / 2$, where $n$ is a positive integer. If the function $p(z)=1+p_{n} z^{n}+\cdots$ is analytic in $\mathbb{U}$ and

$$
\operatorname{Re}\left\{H\left(p(z), z p^{\prime}(z)\right)\right\}>0 \quad(z \in \mathbb{U}),
$$

then $\operatorname{Re}\{p(z)\}>0$ in $\mathbb{U}$.

Lemma 1.5 (see [16]). Let $\beta, \gamma \in \mathbb{C}$ with $\beta \neq 0$ and let $h \in \mathscr{H}(\mathbb{U})$ with $h(0)=c$. If $\operatorname{Re}\{\beta h(z)+\gamma\}>$ $0(z \in \mathbb{U})$, then the solution of the differential equation:

$$
q(z)+\frac{z q^{\prime}(z)}{\beta q(z)+\gamma}=h(z) \quad(z \in \mathbb{U} ; q(0)=c)
$$

is analytic in $\mathbb{U}$ and satisfies $\operatorname{Re}\{\beta q(z)+\gamma\}>0(z \in \mathbb{U})$. 
Lemma 1.6 (see [1]). Let $p \in Q$ with $p(0)=a$ and let $q(z)=a+a_{n} z^{n}+\cdots$ be analytic in $\mathbb{U}$ with $q(z) \not \equiv a$ and $n \geq 1$. If $q$ is not subordinate to $p$, then there exist points $z_{0}=r_{0} e^{i \theta} \in \mathbb{U}$ and $\zeta_{0} \in$ $\partial \mathbb{U} \backslash E(f)$, for which $q\left(\mathbb{U}_{r_{0}}\right) \subset p(\mathbb{U})$,

$$
p\left(z_{0}\right)=q\left(\zeta_{0}\right), \quad z_{0} p^{\prime}\left(z_{0}\right)=m \zeta_{0} q^{\prime}\left(\zeta_{0}\right) \quad(m \geq n) .
$$

Lemma 1.7 (see [2]). Let $q \in \mathscr{L}[a, 1]$, let $\varphi: \mathbb{C}^{2} \rightarrow \mathbb{C}$, and set $\varphi\left(q(z), z q^{\prime}(z)\right) \equiv h(z)$. If $L(z, t)=$ $\varphi\left(q(z), t z q^{\prime}(z)\right)$ is a subordination chain and $p \in \mathscr{H}[a, 1] \cap \mathcal{Q}$, then

$$
h(z) \prec \varphi\left(p(z), z p^{\prime}(z)\right) \quad(z \in \mathbb{U})
$$

implies that

$$
q(z) \prec p(z) \quad(z \in \mathbb{U}) .
$$

Furthermore, if $\varphi\left(q(z), z p^{\prime}(z)\right)=h(z)$ has a univalent solution $q \in Q$, then $q$ is the best subordinant.

A function $L(z, t)$ defined on $\mathbb{U} \times[0, \infty)$ is the subordination chain (or Löwner chain) if $L(\cdot, t)$ is analytic and univalent in $\mathbb{U}$ for all $t \in[0, \infty) ; L(z, \cdot)$ is continuously differentiable on $[0, \infty)$ for all $z \in \mathbb{U}$ and $L(z, s)<L(z, t)$ for $z \in \mathbb{U}$ and $0 \leq s<t$.

Lemma 1.8 (see [17]). The function $L(z, t)=a_{1}(t) z+\cdots$ with $a_{1}(t) \neq 0$ and $\lim _{t \rightarrow \infty}\left|a_{1}(t)\right|=\infty$. Suppose that $L(\cdot ; t)$ ia analytic in $\mathbb{U}$ for all $t \geq 0, L(z ; \cdot)$ is continuously differentiable on $[0, \infty)$ for all $z \in \mathbb{U}$. If $L(z ; t)$ satisfies

$$
|L(z ; t)| \leq K_{0}\left|a_{1}(t)\right| \quad\left(|z|<r_{0}<1 ; 0 \leq t<\infty\right)
$$

for some positive constants $K_{0}$ and $r_{0}$ and

$$
\mathfrak{R}\left\{\frac{z \partial L(z, t) / \partial z}{\partial L(z, t) / \partial t}\right\}>0 \quad(z \in \mathbb{U} ; 0 \leq t<\infty),
$$

then $L(z ; t)$ is a subordination chain.

\section{Main Results}

Firstly, we begin by proving the following subordination theorem involving the multiplier transformation $I_{\mathcal{C}}^{\lambda, \mu}$ defined by (1.10).

Theorem 2.1. Let $f, g \in \mathcal{A}$. Suppose that

$$
\operatorname{Re}\left\{1+\frac{z \phi^{\prime \prime}(z)}{\phi^{\prime}(z)}\right\}>-\delta \quad\left(\phi(z):=(1-\alpha) I_{c}^{\lambda+1, \mu} g(z)+\alpha I_{c}^{\lambda, \mu} g(z) ; 0 \leq \alpha<1 ; z \in \mathbb{U}\right),
$$


where

$$
\delta=\frac{(1-\alpha)^{2}+|c-1+\alpha|^{2}-\left|(1-\alpha)^{2}-(c-1+\alpha)^{2}\right|}{4(1-\alpha) \operatorname{Re}\{c-1+\alpha\}} \quad(\operatorname{Re}\{c-1+\alpha\}>0)
$$

Then the subordination:

$$
(1-\alpha) I_{c}^{\lambda+1, \mu} f(z)+\alpha I_{c}^{\lambda, \mu} f(z) \prec \phi(z) \quad(z \in \mathbb{U})
$$

implies that

$$
I_{c}^{\lambda, \mu} f(z) \prec I_{c}^{\lambda, \mu} g(z) \quad(z \in \mathbb{U})
$$

Moreover, the function $I_{c}^{\lambda, \mu} g(z)$ is the best dominant.

Proof. Let us define the functions $F$ and $G$, respectively, by

$$
F(z):=I_{\mathcal{c}}^{\lambda, \mu} f(z), \quad G(z):=I_{c}^{\lambda, \mu} g(z)
$$

We first show that if the function $q$ is defined by

$$
q(z):=1+\frac{z G^{\prime \prime}(z)}{G^{\prime}(z)} \quad(z \in \mathbb{U}),
$$

then

$$
\operatorname{Re}\{q(z)\}>0 \quad(z \in \mathbb{U})
$$

Taking the logarithmic differentiation on both sides of the second equation in (2.5) and using (1.11) for $g \in \mathcal{A}$, we obtain

$$
c \phi(z)=(c-1+\alpha) G(z)+(1-\alpha) z G^{\prime}(z)
$$

which, in conjunction with (2.8), yields the relationship:

$$
\begin{aligned}
1+\frac{z \phi^{\prime \prime}(z)}{\phi^{\prime}(z)} & =1+\frac{z G^{\prime \prime}(z)}{G^{\prime}(z)}+\frac{z q^{\prime}(z)}{q(z)+(c-1+\alpha) /(1-\alpha)} \\
& =q(z)+\frac{z q^{\prime}(z)}{q(z)+(c-1+\alpha) /(1-\alpha)} \equiv h(z) .
\end{aligned}
$$

From (2.1), we have

$$
\operatorname{Re}\left\{h(z)+\frac{c-1+\alpha}{1-\alpha}\right\}>0 \quad(z \in \mathbb{U})
$$


and by using Lemma 1.5, we conclude that the differential equation (2.9) has a solution $q \in$ $\mathscr{H}(\mathbb{U})$ with $q(0)=h(0)=1$.

Let us put

$$
H(u, v)=u+\frac{v}{u+(c-1+\alpha) /(1-\alpha)}+\delta
$$

where $\delta$ is given by (2.2). From (2.1), (2.9), and (2.11), we obtain

$$
\operatorname{Re}\left\{H\left(q(z), z q^{\prime}(z)\right)\right\}>0 \quad(z \in \mathbb{U}) .
$$

Now we proceed to show that $\operatorname{Re}\{H(i s, t)\} \leq 0$ for all real $s$ and $t \leq-\left(1+s^{2}\right) / 2$. From (2.11), we have

$$
\begin{aligned}
\operatorname{Re}\{H(i s, t)\} & =\operatorname{Re}\left\{i s+\frac{t}{i s+(c-1+\alpha) /(1-\alpha)}+\delta\right\} \\
& =\frac{t(c-1+\alpha) /(1-\alpha)}{|(c-1+\alpha) /(1-\alpha)+i s|^{2}}+\delta \\
& \leq-\frac{E_{\delta}(s)}{2|(c-1+\alpha) /(1-\alpha)+i s|^{2}}
\end{aligned}
$$

where

$$
\begin{aligned}
E_{\delta}(s):= & \left(\frac{\operatorname{Re}\{c-1+\alpha\}}{(1-\alpha)}-2 \delta\right) s^{2}-\frac{4 \delta s \operatorname{Im}\{c-1+\alpha\}}{(1-\alpha)} \\
& -2 \delta\left|\frac{(c-1+\alpha)}{(1-\alpha)}\right|^{2}+\frac{\operatorname{Re}\{c-1+\alpha\}}{(1-\alpha)} .
\end{aligned}
$$

For $\delta$ given by (2.2), we can prove easily that the expression $E_{\delta}(s)$ given by (2.14) is positive or equal to zero. Moreover, the quadratic expression by $s$ in (2.14) is a perfect square for the assumed value of $\delta$. Hence from (2.13), we see that $\operatorname{Re}\{H(i s, t)\} \leq 0$ for all real $s$ and $t \leq-\left(1+s^{2}\right) / 2$. Thus, by using Lemma 1.4 , we conclude that $\operatorname{Re}\{q(z)\}>0$ for all $z \in \mathbb{U}$. That is, $q$ is convex in $\mathbb{U}$.

Next, we prove that the subordination condition (2.3) implies that

$$
F(z) \prec G(z) \quad(z \in \mathbb{U})
$$

for the functions $F$ and $G$ defined by (2.5). Without loss of generality, we can assume that $G$ is analytic and univalent on $\overline{\mathbb{U}}$ and that $G^{\prime}(\zeta) \neq 0(|\zeta|=1)$. Now we consider the function $L(z, t)$ given by

$$
L(z, t):=\frac{c-1+\alpha}{c} G(z)+\frac{(1-\alpha)(1+t)}{c} z G^{\prime}(z) \quad(z \in \mathbb{U} ; 0 \leq t<\infty) .
$$

We note that 
Abstract and Applied Analysis

$$
\left.\frac{\partial L(z, t)}{\partial z}\right|_{z=0}=G^{\prime}(0)\left(\frac{(c-1+\alpha)+(1-\alpha)(1+t)}{c}\right) \neq 0 \quad(0 \leq t<\infty ; \operatorname{Re}\{c-1+\alpha\}>0) .
$$

This shows that the function

$$
L(z, t)=a_{1}(t) z+\cdots
$$

satisfies the condition $a_{1}(t) \neq 0$ for all $t \in[0, \infty)$. By using the well-known growth and distortion theorems for convex functions, it is easy to check that the first part of Lemma 1.8 is satisfied. Furthermore, we have

$$
\operatorname{Re}\left\{\frac{z \partial L(z, t) / \partial z}{\partial L(z, t) / \partial t}\right\}=\operatorname{Re}\left\{\frac{c-1+\alpha}{1-\alpha}+(1+t)\left(1+\frac{z G^{\prime \prime}(z)}{G^{\prime}(z)}\right)\right\}>0
$$

since $G$ is convex and $\operatorname{Re}\{c-1+\alpha\}>0$. Therefore, by virtue of Lemma $1.8, L(z, t)$ is a subordination chain. We observe from the definition of a subordination chain that

$$
\begin{gathered}
\phi(z)=\frac{c-1+\alpha}{c} G(z)+\frac{1-\alpha}{c} z G^{\prime}(z)=L(z, 0), \\
L(z, 0) \prec L(z, t) \quad(z \in \mathbb{U} ; 0 \leq t<\infty) .
\end{gathered}
$$

This implies that

$$
L(\zeta, t) \notin L(\mathbb{U}, 0)=\phi(\mathbb{U}) \quad(\zeta \in \partial \mathbb{U} ; 0 \leq t<\infty)
$$

Now suppose that $F$ is not subordinate to $G$, then by Lemma 1.6, there exists points $z_{0} \in \mathbb{U}$ and $\zeta_{0} \in \partial \mathbb{U}$ such that

$$
F\left(z_{0}\right)=G\left(\zeta_{0}\right), \quad z_{0} F\left(z_{0}\right)=(1+t) \zeta_{0} G^{\prime}\left(\zeta_{0}\right) \quad(0 \leq t<\infty) .
$$

Hence we have

$$
\begin{aligned}
L\left(\zeta_{0}, t\right) & =\frac{c-1+\alpha}{c} G\left(\zeta_{0}\right)+\frac{(1-\alpha)(1+t)}{c} \zeta_{0} G^{\prime}\left(\zeta_{0}\right) \\
& =\frac{c-1+\alpha}{c} F\left(z_{0}\right)+\frac{1-\alpha}{c} z_{0} F^{\prime}\left(z_{0}\right) \\
& =(1-\alpha) I_{c}^{\lambda+1, \mu} f\left(z_{0}\right)+\alpha I_{c}^{\lambda, \mu} f\left(z_{0}\right) \in \phi(\mathbb{U}),
\end{aligned}
$$

by virtue of the subordination condition (2.3). This contracts the above observation that $L\left(\zeta_{0}, t\right) \notin \phi(\mathbb{U})$. Therefore, the subordination condition (2.3) must imply the subordination given by (2.15). Considering $F(z)=G(z)$, we see that the function $G(z)$ is the best dominant. This evidently completes the proof of Theorem 2.1.

Remark 2.2. We note that $\delta$ given by (2.2) in Theorem 2.1 satisfies the inequality $0<\delta \leq 1 / 2$. 
We next prove a dual problem of Theorem 2.1, in the sense that the subordinations are replaced by superordinations.

Theorem 2.3. Let $f, g \in \mathcal{A}$. Suppose that

$$
\operatorname{Re}\left\{1+\frac{z \phi^{\prime \prime}(z)}{\phi^{\prime}(z)}\right\}>-\delta \quad\left(\phi(z):=(1-\alpha) I_{c}^{\lambda+1, \mu} g(z)+\alpha I_{c}^{\lambda, \mu} g(z) ; 0 \leq \alpha<1 ; z \in \mathbb{U}\right)
$$

where $\delta$ is given by (2.2), and the function $I_{c}^{\lambda+1, \mu} f(z)$ is univalent in $\mathbb{U}$ and $I_{c}^{\lambda, \mu} f(z) \in Q$. Then the superordination:

$$
\phi(z) \prec(1-\alpha) I_{c}^{\lambda+1, \mu} f(z)+\alpha I_{c}^{\lambda, \mu} f(z) \quad(z \in \mathbb{U})
$$

implies that

$$
I_{c}^{\lambda, \mu} g(z) z \prec I_{c}^{\lambda, \mu} f(z) \quad(z \in \mathbb{U})
$$

Moreover, the function $I_{c}^{\lambda, \mu} g(z)$ is the best subordinant.

Proof. Let us define the functions $F$ and $G$, respectively, by (2.5). We first note that, if the function $q$ is defined by (2.6), by using (2.8), then we obtain

$$
\begin{aligned}
\phi(z) & =\frac{c-1+\alpha}{c} G(z)+\frac{1-\alpha}{c} z G^{\prime}(z) \\
& =: \varphi\left(G(z), z G^{\prime}(z)\right) .
\end{aligned}
$$

After a simple calculation, (2.27) yields the relationship:

$$
1+\frac{z \phi^{\prime \prime}(z)}{\phi^{\prime}(z)}=q(z)+\frac{z q^{\prime}(z)}{q(z)+(c-1+\alpha) /(1-\alpha)} .
$$

Then by using the same method as in the proof of Theorem 2.1, we can prove that $\operatorname{Re}\{q(z)\}>$ 0 for all $z \in \mathbb{U}$. That is, $G$ defined by (2.6) is convex (univalent) in $\mathbb{U}$.

Next, we prove that the subordination condition (2.27) implies that

$$
F(z) \prec G(z) \quad(z \in \mathbb{U})
$$

for the functions $F$ and $G$ defined by (2.5). Now consider the function $L(z, t)$ defined by

$$
L(z, t):=\frac{c-1+\alpha}{c} G(z)+\frac{(1-\alpha) t}{c} z G^{\prime}(z) \quad(z \in \mathbb{U} ; 0 \leq t<\infty) .
$$

Since $G$ is convex and $\operatorname{Re}\{c-1+\alpha\}>0$, we can prove easily that $L(z, t)$ is a subordination chain as in the proof of Theorem 2.1. Therefore according to Lemma 1.7, we conclude that the 
superordination condition (2.27) must imply the superordination given by (2.29). Furthermore, since the differential equation (2.27) has the univalent solution $G$, it is the best subordinant of the given differential superordination. Therefore we complete the proof of Theorem 2.3. theorem.

If we combine this Theorems 2.1 and 2.3, then we obtain the following sandwich-type

Theorem 2.4. Let $f, g_{k} \in \mathcal{A}(k=1,2)$. Suppose that

$$
\operatorname{Re}\left\{1+\frac{z \phi_{k}^{\prime \prime}(z)}{\phi_{k}^{\prime}(z)}\right\}>-\delta \quad\left(\phi_{k}(z):=(1-\alpha) I_{c}^{\lambda+1, \mu} g_{k}(z)+\alpha I_{c}^{\lambda, \mu} g_{k}(z) ; k=1,2 ; 0 \leq \alpha<1 ; z \in \mathbb{U}\right),
$$

where $\delta$ is given by (2.2), and the function $I_{c}^{\lambda+1, \mu} f(z)$ is univalent in $\mathbb{U}$ and $I_{c}^{\lambda, \mu} f(z) \in \mathcal{Q}$. Then the subordination relation:

$$
\phi_{1}(z) \prec I_{c}^{\lambda+1, \mu} f(z) \prec \phi_{2}(z) \quad(z \in \mathbb{U})
$$

implies that

$$
I_{c}^{\lambda, \mu} g_{k}(z) \prec I_{c}^{\lambda, \mu} f(z) \prec I_{c}^{\lambda, \mu} g_{2}(z) \quad(z \in \mathbb{U})
$$

Moreover, the functions $I_{c}^{\lambda, \mu} g_{1}(z)$ and $I_{c}^{\lambda, \mu} g_{2}(z)$ are the best subordinant and the best dominant, respectively.

The assumption of Theorem 2.4, that the functions $I_{c}^{\lambda+1, \mu} f(z)$ and $I_{c}^{\lambda, \mu} f(z)$ need to be univalent in $\mathbb{U}$, may be replaced by another conditions in the following result.

Corollary 2.5. Let $f, g_{k} \in \mathcal{A}(k=1,2)$. Suppose that the condition (2.31) is satisfied and

$$
\operatorname{Re}\left\{1+\frac{z \psi^{\prime \prime}(z)}{\psi^{\prime}(z)}\right\}>-\delta \quad\left(\psi(z):=(1-\alpha) I_{c}^{\lambda+1, \mu} f(z)+\alpha I_{c}^{\lambda, \mu} f(z) ; 0 \leq \alpha<1 ; z \in \mathbb{U} ; f \in Q\right),
$$

where $\delta$ is given by (2.2). Then the subordination relation:

$$
\phi_{1}(z) \prec \psi(z) \prec \phi_{2}(z) \quad(z \in \mathbb{U})
$$

implies that

$$
I_{c}^{\lambda, \mu} g_{k}(z) \prec I_{c}^{\lambda, \mu} f(z) \prec I_{c}^{\lambda, \mu} g_{2}(z) \quad(z \in \mathbb{U})
$$

Moreover, the functions $I_{c}^{\lambda, \mu} g_{1}(z)$ and $I_{c}^{\lambda, \mu} g_{2}(z)$ are the best subordinant and the best dominant, respectively. 
Proof. In order to prove Corollary 2.5, we have to show that the condition (2.34) implies the univalence of $\psi(z)$ and $F(z):=I_{c}^{\lambda, \mu} f(z)$. Since $0<\delta \leq 1 / 2$ from Remark 2.2, the condition (2.34) means that $\psi$ is a close-to-convex function in $\mathbb{U}$ (see [18]) and hence $\psi$ is univalent in $\mathbb{U}$. Furthermore, by using the same techniques as in the proof of Theorem 2.4, we can prove the convexity (univalence) of $F$ and so the details may be omitted. Therefore, by applying Theorem 2.4, we obtain Corollary 2.5.

By setting $c=2$ and $\alpha=0$ in Theorem 2.4 , so that $\delta=1 / 2$, we deduce the following consequence of Theorem 2.4.

Corollary 2.6. Let $f, g_{k} \in \mathcal{A}(k=1,2)$. Suppose that

$$
\operatorname{Re}\left\{1+\frac{z \phi_{k}^{\prime \prime}(z)}{\phi_{k}^{\prime}(z)}\right\}>-\frac{1}{2}\left(\phi_{k}(z):=I_{2}^{\lambda+1, \mu} g_{k}(z) ; k=1,2 ; z \in \mathbb{U}\right),
$$

and the function $I_{2}^{\lambda+1, \mu} f(z)$ is univalent functions in $\mathbb{U}$ and $I_{2}^{\lambda, \mu} f(z) \in \mathcal{Q}$. Then the subordination relation:

$$
I_{2}^{\lambda+1, \mu} g_{1}(z) \prec I_{2}^{\lambda+1, \mu} f(z) \prec I_{2}^{\lambda+1, \mu} g_{2}(z) \quad(z \in \mathbb{U})
$$

implies that

$$
I_{2}^{\lambda, \mu} g_{1}(z) \prec I_{2}^{\lambda, \mu} f(z) \prec I_{2}^{\lambda, \mu} g_{2}(z) \quad(z \in \mathbb{U})
$$

Moreover, the functions $I_{2}^{\lambda, \mu} g_{1}(z)$ and $I_{2}^{\lambda, \mu} g_{2}(z)$ are the best subordinant and the best dominant, respectively. result.

If we take $c=2+i$ and $\alpha=0$ in Theorem 2.4, then we easily to lead to the following

Corollary 2.7. Let $f, g_{k} \in \mathcal{A}(k=1,2)$. Suppose that

$$
\operatorname{Re}\left\{1+\frac{z \phi_{k}^{\prime \prime}(z)}{\phi_{k}^{\prime}(z)}\right\}>-\frac{3-\sqrt{5}}{4} \quad\left(\phi_{k}(z):=I_{2+i}^{\lambda, \mu} g_{k}(z) ; k=1,2 ; z \in \mathbb{U}\right),
$$

and the function $I_{2+i}^{\lambda+1, \mu} f(z)$ is univalent functions in $\mathbb{U}$ and $I_{2+i}^{\lambda, \mu} f(z) \in Q$. Then the subordination relation:

$$
I_{2+i}^{\lambda+1, \mu} g_{1}(z) \prec I_{2+i}^{\lambda+1, \mu} f(z) \prec I_{2+i}^{\lambda+1, \mu} g_{2}(z) \quad(z \in \mathbb{U})
$$

implies that

$$
I_{2+i}^{\lambda, \mu} g_{1}(z) \prec I_{2+i}^{\lambda, \mu} f(z) \prec I_{2+i}^{\lambda, \mu} g_{2}(z) \quad(z \in \mathbb{U})
$$


Moreover, the functions $I_{2+i}^{\lambda, \mu} g_{1}(z)$ and $I_{2+i}^{\lambda, \mu} g_{2}(z)$ are the best subordinant and the best dominant, respectively.

The proof of Theorem 2.8 below is similar to that of Theorem 2.4 by using (1.12) and so the details may be omitted.

Theorem 2.8. Let $f, g_{k} \in \mathcal{A}(k=1,2)$. Suppose that

$$
\operatorname{Re}\left\{1+\frac{z \phi_{k}^{\prime \prime}(z)}{\phi_{k}^{\prime}(z)}\right\}>-\delta \quad\left(\phi_{k}(z):=(1-\alpha) I_{c}^{\lambda, \mu+1} g_{k}(z)+\alpha I_{c}^{\lambda, \mu} g_{k}(z) ; k=1,2 ; 0 \leq \alpha<1 ; z \in \mathbb{U}\right),
$$

where $\delta$ is given by (2.2) with $c=\mu$, and the function $I_{c}^{\lambda, \mu+1} f(z)$ is univalent in $\mathbb{U}$ and $I_{c}^{\lambda, \mu} f(z) \in \mathcal{Q}$. Then the subordination relation:

$$
\phi_{1}(z) \prec I_{c}^{\lambda, \mu+1} f(z) \prec \phi_{2}(z) \quad(z \in \mathbb{U})
$$

implies that

$$
I_{c}^{\lambda, \mu} g_{k}(z) \prec I_{c}^{\lambda, \mu} f(z) \prec I_{c}^{\lambda, \mu} g_{2}(z) \quad(z \in \mathbb{U})
$$

Moreover, the functions $I_{c}^{\lambda, \mu} g_{1}(z)$ and $I_{c}^{\lambda, \mu} g_{2}(z)$ are the best subordinant and the best dominant, respectively.

By using a similar method given in the proof of Theorems 2.4 and 2.8, we have the corresponding two theorems below.

Theorem 2.9. Let $f, g_{k} \in \mathcal{A}(k=1,2)$ with the additional condition $g_{k}^{\prime \prime}(z) \neq 0$. Suppose that

$$
\begin{aligned}
\operatorname{Re}\{1 & \left.+\frac{z \phi_{k}^{\prime \prime}(z)}{\phi_{k}^{\prime}(z)}\right\} \\
& >-\delta\left(\phi_{k}(z):=\frac{(1-\alpha) I_{c}^{\lambda+1, \mu} g_{k}(z)+\alpha I_{c}^{\lambda, \mu} g_{k}(z)}{z} ; k=1,2 ; 0 \leq \alpha<1 ; z \in \mathbb{U}\right),
\end{aligned}
$$

where $\delta$ is given by

$$
\delta=\frac{(1-\alpha)^{2}+|c|^{2}-\left|(1-\alpha)^{2}-c^{2}\right|}{4(1-\alpha) \operatorname{Re}\{c\}} \quad(\operatorname{Re}\{c\}>0)
$$

and the function $I_{c}^{\lambda+1, \mu} f(z)$ is univalent in $\mathbb{U}$ and $I_{c}^{\lambda, \mu} f(z) \in Q$. Then the subordination relation:

$$
\phi_{1}(z) \prec \frac{I_{c}^{\lambda+1, \mu} f(z)}{z} \prec \phi_{2}(z) \quad(z \in \mathbb{U})
$$


implies that

$$
\frac{I_{c}^{\lambda, \mu} g_{1}(z)}{z} \prec \frac{I_{c}^{\lambda, \mu} f(z)}{z} \prec \frac{I_{c}^{\lambda, \mu} g_{2}(z)}{z} \quad(z \in \mathbb{U})
$$

Moreover, the functions $I_{c}^{\lambda, \mu} g_{1}(z) / z$ and $I_{c}^{\lambda, \mu} g_{2}(z) / z$ are the best subordinant and the best dominant, respectively.

Theorem 2.10. Let $f, g_{k} \in \mathcal{A}(k=1,2)$ with the additional condition $g_{k}^{\prime \prime}(z) \neq 0$. Suppose that

$$
\begin{aligned}
& \operatorname{Re}\left\{1+\frac{z \phi_{k}^{\prime \prime}(z)}{\phi_{k}^{\prime}(z)}\right\} \\
& \quad>-\delta\left(\phi_{k}(z):=\frac{(1-\alpha) I_{c}^{\lambda, \mu+1} g_{k}(z)+\alpha I_{c}^{\lambda, \mu} g_{k}(z)}{z} ; k=1,2 ; 0 \leq \alpha<1 ; z \in \mathbb{U}\right),
\end{aligned}
$$

where $\delta$ is given by (2.47) with $c=\mu$, and the function $I_{c}^{\lambda, \mu+1} f(z) / z$ is univalent in $\mathbb{U}$ and $I_{c}^{\lambda, \mu}$ $f(z) / z \in Q$. Then the subordination relation:

$$
\phi_{1}(z) \prec \frac{I_{c}^{\lambda, \mu+1} f(z)}{z} \prec \phi_{2}(z) \quad(z \in \mathbb{U})
$$

implies that

$$
\frac{I_{c}^{\lambda, \mu} g_{1}(z)}{z} \prec \frac{I_{c}^{\lambda, \mu} f(z)}{z} \prec \frac{I_{c}^{\lambda, \mu} g_{2}(z)}{z} \quad(z \in \mathbb{U})
$$

Moreover, the functions $I_{c}^{\lambda, \mu} g_{1}(z) / z$ and $I_{c}^{\lambda, \mu} g_{2}(z) / z$ are the best subordinant and the best dominant, respectively.

\section{Acknowledgment}

This research was supported by the Basic Science Research Program through the National Research Foundation of Korea(NRF) funded by the Ministry of Education, Science and Technology (no. 2011-0007037).

\section{References}

[1] S. S. Miller and P. T. Mocanu, Differential Subordinations, Theory and Application, vol. 225 of Monographs and Textbooks in Pure and Applied Mathematics, Marcel Dekker Inc., New York, NY, USA, 2000.

[2] S. S. Miller and P. T. Mocanu, "Subordinants of differential superordinations," Complex Variables. Theory and Application, vol. 48, no. 10, pp. 815-826, 2003.

[3] Y. Komatu, Distortion Theorems in Relation to Linear Integral Operators, vol. 385 of Mathematics and its Applications, Kluwer Academic Publishers, Dordrecht, The Netherlands, 1996. 
[4] T. M. Flett, "The dual of an inequality of Hardy and Littlewood and some related inequalities," Journal of Mathematical Analysis and Applications, vol. 38, pp. 746-765, 1972.

[5] I. B. Jung, Y. C. Kim, and H. M. Srivastava, "The Hardy space of analytic functions associated with certain one-parameter families of integral operators," Journal of Mathematical Analysis and Applications, vol. 176, no. 1, pp. 138-147, 1993.

[6] J.-L. Liu, "A linear operator and strongly starlike functions," Journal of the Mathematical Society of Japan, vol. 54, no. 4, pp. 975-981, 2002.

[7] K. I. Noor, "On new classes of integral operators," Journal of Natural Geometry, vol. 16, no. 1-2, pp. 71-80, 1999.

[8] J. H. Choi, M. Saigo, and H. M. Srivastava, "Some inclusion properties of a certain family of integral operators," Journal of Mathematical Analysis and Applications, vol. 276, no. 1, pp. 432-445, 2002.

[9] J.-L. Liu, "The Noor integral and strongly starlike functions," Journal of Mathematical Analysis and Applications, vol. 261, no. 2, pp. 441-447, 2001.

[10] J.-L. Liu and K. I. Noor, "Some properties of Noor integral operator," Journal of Natural Geometry, vol. 21, no. 1-2, pp. 81-90, 2002.

[11] K. I. Noor and M. A. Noor, "On integral operators," Journal of Mathematical Analysis and Applications, vol. 238, no. 2, pp. 341-352, 1999.

[12] S. S. Miller, P. T. Mocanu, and M. O. Reade, "Subordination-preserving integral operators," Transactions of the American Mathematical Society, vol. 283, no. 2, pp. 605-615, 1984.

[13] T. Bulboacă, "Integral operators that preserve the subordination," Bulletin of the Korean Mathematical Society, vol. 34, no. 4, pp. 627-636, 1997.

[14] T. Bulboacă, "A class of superordination-preserving integral operators," Indagationes Mathematicae. New Series, vol. 13, no. 3, pp. 301-311, 2002.

[15] S. S. Miller and P. T. Mocanu, "Differential subordinations and univalent functions," The Michigan Mathematical Journal, vol. 28, no. 2, pp. 157-172, 1981.

[16] S. S. Miller and P. T. Mocanu, "Univalent solutions of Briot-Bouquet differential equations," Journal of Differential Equations, vol. 56, no. 3, pp. 297-309, 1985.

[17] C. Pommerenke, Univalent functions, Vandenhoeck \& Ruprecht, Göttingen, Germany, 1975.

[18] W. Kaplan, "Close-to-convex schlicht functions," The Michigan Mathematical Journal, vol. 1, pp. 169$185,1952$. 


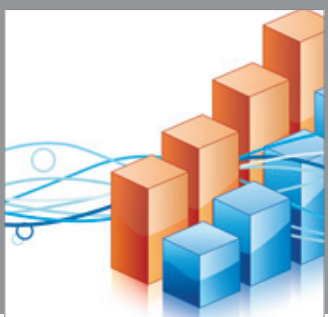

Advances in

Operations Research

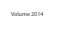

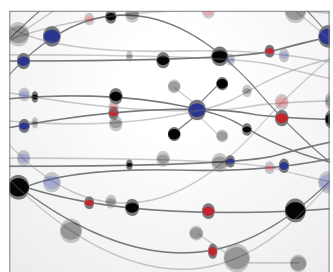

\section{The Scientific} World Journal
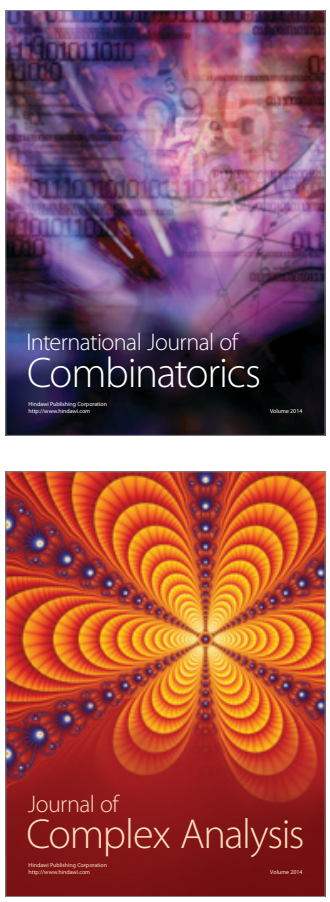

International Journal of

Mathematics and

Mathematical

Sciences
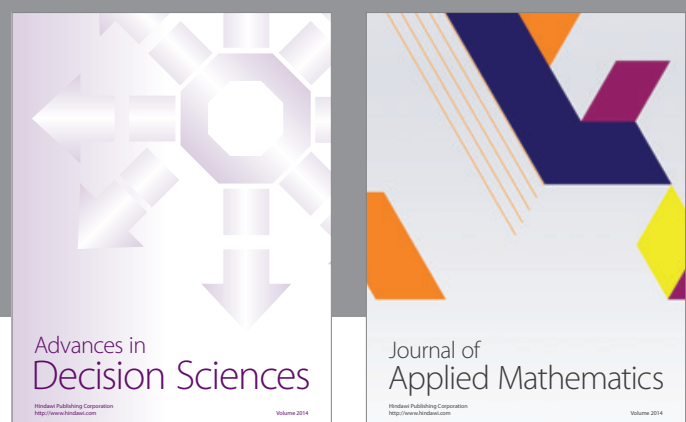

Journal of

Applied Mathematics
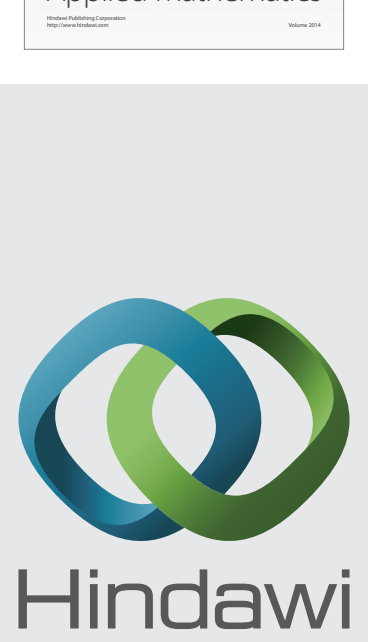

Submit your manuscripts at http://www.hindawi.com
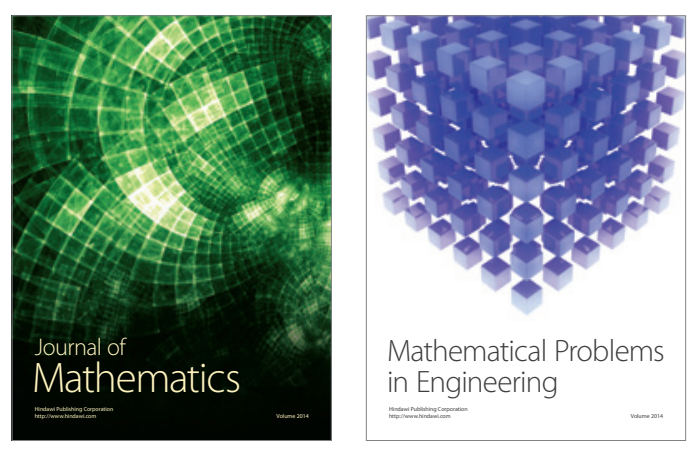

Mathematical Problems in Engineering
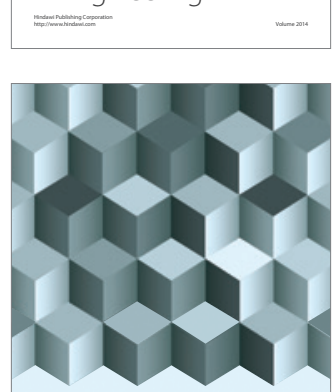

Journal of

Function Spaces
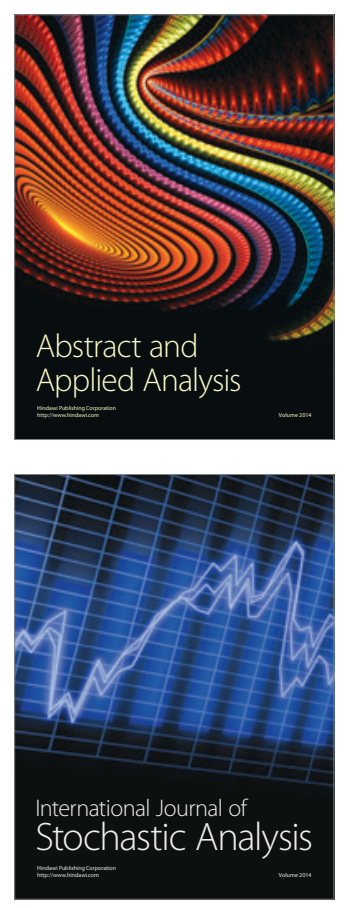

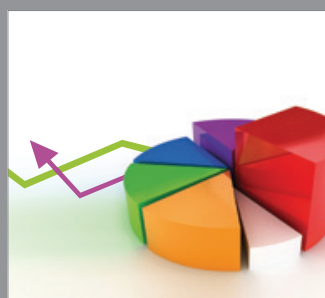

ournal of

Probability and Statistics

Promensencen
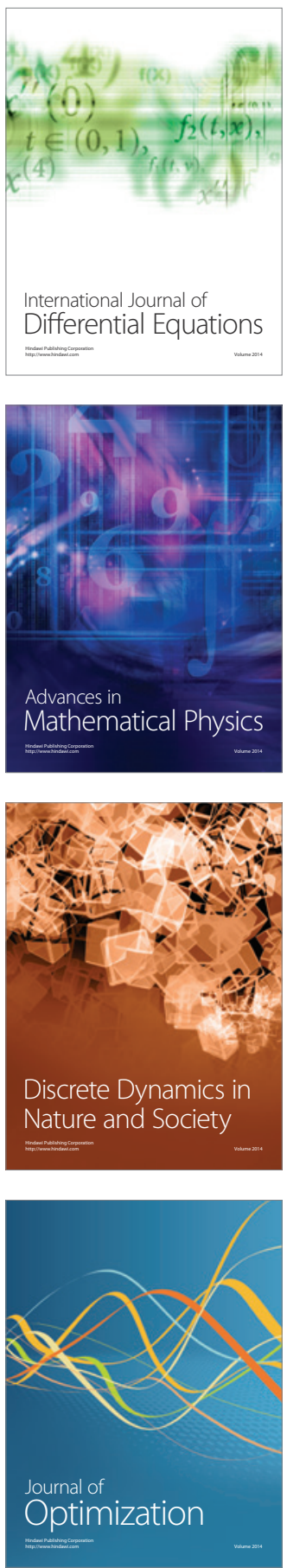